\title{
La gestión directiva como potenciadora de la mejora del clima organizacional y la convivencia en las instituciones de educación media superior
}

The directive management as an enhancer of the improvement of the organizational climate and coexistence in high school

\section{A gestãodiretiva como potenciador da melhoria do clima organizacional e da coexistêncianasinstituições do ensinosecundário superior}

Evangelina López Ramírez

Universidad Autónoma de Baja California, México evangelinalopez@uabc.edu.mx https://orcid.org/0000-0003-0960-1788

Luis Fernando García Hernández Universidad Autónoma de Baja California, México luis.fernando.garcia.hernandez@uabc.edu.mx https://orcid.org/0000-0003-4704-1662

Jorge Eduardo Martínez Iñiguez Universidad Autónoma de Baja California, México jorge.martinez43@uabc.edu.mx https://orcid.org/0000-0002-8833-5600

\section{Resumen}

Este documento tiene como objetivo destacar la función que realizan los directores y equipos directivos de instituciones educativas de nivel medio superior en el plano específico de la gestión del clima organizacional y la convivencia con los diferentes actores que coinciden en la escuela. Para ello, se han tomado en cuenta las apreciaciones valorativas delos profesores de dichos centros educativos en torno al desempeño de sus directivos. La metodología empleada se sustentó en la investigación cualitativa, pues se procuró recabar información sobre el tema de estudio a través de un cuestionario y unas escalas de tipo Likert. Los principales resultados indican que el profesorado está en 

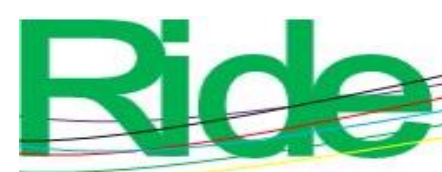

Revista Iberoamericana para la

Investigación y el Desarrollo Educativo

ISSN $2007-7467$

desacuerdo con la manera en que sus directivos ejercen su liderazgo y los trabajos de gestión institucional, lo que se vincula con negativos resultados de aprendizaje en los estudiantes. En conclusión, se considera importante que los directivos impulsen el trabajo colaborativo y la comunicación dinámica y abierta en las instituciones de educación media superior para resolver sus problemascontextuales más apremiantes.

Palabras clave:clima organizacional, convivencia, equipos directivos.

\begin{abstract}
The purpose of this document is to highlight the role of directors and management teams in upper secondary educational institutions in the specific field of organizational climate management and coexistence with the different actors that coincide in the school. For this, the evaluative evaluations of the teachers of said educational centers have been taken into account regarding the performance of their managers. The methodology used was based on qualitative research, since it was sought to gather information about the study topic through a questionnaire and Likert type scales. The main results indicate that the teaching staff disagrees with the way in which their managers exercise their leadership and institutional management work, which is linked to negative learning outcomes in the students. In conclusion, it is considered important that managers promote collaborative work and dynamic and open communication in higher education institutions to solve their most pressing contextual problems.
\end{abstract}

Keywords: organizational climate, coexistence, management teams.

\title{
Resumo
}

O objetivo deste documento é destacar o papel dos diretores e das equipes de gestãonasinstituições de ensinomédio no campo específico da gestão do clima organizacional e da coexistênciacom os diferentes atores que coincidemnaescola. Para tanto, os juízos de valor dos professoresdesses centros de ensinoforam levados em conta em relaçãoaodesempenho de seus gestores. A metodologia utilizada baseou-se em pesquisa qualitativa, poisbuscou-se coletarinformações sobre o tema do estudo por meio de 
questionário e escalas do tipo Likert. Os principais resultados indicam que o corpo docente discorda da maneira como seus gerentes exercemseutrabalho de liderança e gestão institucional, o que está vinculado aos resultados negativos da aprendizagem nos alunos. Em conclusão, considera-se importante que os gestores promovam o trabalho colaborativo e a comunicaçãodinâmica e abertanasinstituições de ensino superior para resolver seus problemas contextuaismaisprementes.

Palavras-chave: clima organizacional, coexistência, equipes de gestão.

Fecha Recepción: Junio 2018

Fecha Aceptación: Noviembre 2018

\section{Introducción}

La pretensión de los gobiernos latinoamericanos por atender la formación pertinente de los directivos de instituciones educativas ha evolucionado de manera diferenciada. La Oficina Regional de Educación para América Latina y el Caribe (OREALC), perteneciente a la Organización de las Naciones Unidas para la Educación, la Ciencia y la Cultura (UNESCO, por sus siglas en inglés), informaba que Guatemala era el país con menor porcentaje $(35.4 \%)$ de directivos que había realizado y concluido estudios específicos en dicha área, seguido deMéxico(72.4\%), Cuba (80.9\%), Argentina (89.4\%), Perú (94.9\%) y Chile (97.5 \%)(Murillo, 2012, citado por Weinstein, Hernández, Cuellar yFlessa, 2014).

Esta estrategia se encuentra directamente relacionada con la posibilidad de avanzar en los procesos de reforma e innovación de los sistemas educativos latinoamericanos (Poggi, 2001), pues el papel del directivo es central en la construcción de ambientes organizacionales colaborativos, flexibles, innovadores, implicativos y amables, baluartessustantivos que pueden potenciar la percepción de los actores educativospara inducir la vivencia de condiciones que se constituyan como potentes para la innovación educativa y escolar (Ojeda, 2014, citado por Sandoval, Surdez y Pérez, 2017).

Ciertamente, a través de su gestión los directivosdeben asumir el liderazgo para reconstituir el tejido humano de sus escuelas, sin dejar de lado las demandas de distintos componentes en materia de objetivos, recursos (humanos, materiales y financieros),organización,procedimientos y mecanismos con los que opera sin dejar de lado 


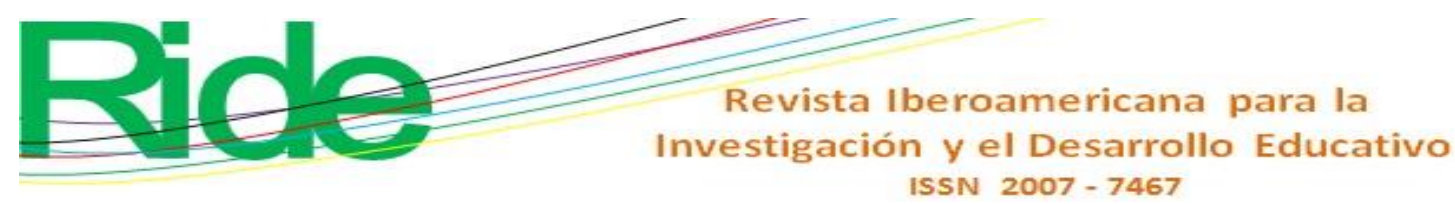

la cultura escolar (valores, creencias o significados compartidos por sus miembros), así como elentorno donde se ubica (Antúnez, 1993, citado por el Instituto Nacional para la Evaluación de la Educación [INEE], s.f.), todo esto con el propósito de que los alumnos consigan resultados académicos positivos (Araiza, Magaña y García y Carrillo, 2014).

Explicado lo anterior, se puede indicar que los objetivos del presente estudio son los siguientes: 1)examinar la labor que efectúan directivos y equipos directivos en torno a la gestión del clima organizacional y la convivencia en instituciones de educación media superior; 2)analizar las apreciaciones valorativas del profesorado de educación media superior en relación con las acciones que realizan los directivos y equipos directivos para instaurar una óptima gestión del clima organizacional y convivencia en los plantes del nivel medio superior, y 3) promover nuevos estudiosque permitanfomentaruna cultura organizacional caracterizada por el trabajo colaborativo de todos los actores que conforman una comunidad educativa para afrontar sus problemas contextuales más apremiantes.

Para ello, en este documento se hará referencia a la comprensión de la gestión directiva, el clima organizacional y la convivencia. Posteriormente, se describirá la metodología empleada en la investigación, sus principales resultados,así como la discusión y las conclusiones respectivas.

\section{La gestión directiva}

La funciónde los equiposdirectivos se ha tornado más compleja no solo porque han aumentado sus tareas, sino también porque el nivel de exigencia requerida se elevade forma progresiva. En la actualidad, por ejemplo, es necesario que el directivo cuente con determinados conocimientos, aptitudes y capacidades para poder desarrollar su función oportunamente (Vázquez, Liesa yBernal, 2016).Por eso, cuando se emplea el término gestión implícitamente se está indicando la responsabilidad de organizar a un grupo de personas para alcanzar un fin predeterminado. Sobre el referido vocablo, Cruzata y Rodríguez (2016) explican lo siguiente:

[La gestión] se define como el conjunto de acciones integradas para el logro de un objetivo a cierto plazo; es la acción principal de la administración y es un eslabón intermedio entre la planificación y los objetivos concretos que se pretenden alcanzar (p. 3). 

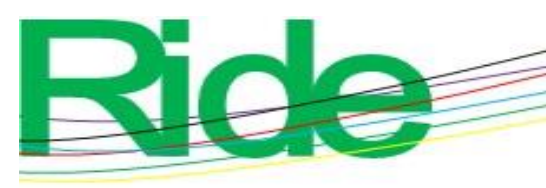

Revista Iberoamericana para la

Investigación y el Desarrollo Educativo

ISSN 2007 - 7467

Ahora bien, en cuanto a la gestión directiva, esta se puede entendercomo "una capacidad de generar una relación adecuada entre la estructura, la estrategia, los sistemas, el estilo, las capacidades, la gente y los objetivos superiores de la organización considerada" (Casasús, 1997,citado por Lópezet al., 2015, p. 18). Esto significa que la gestión directiva impacta en la manera como el plantel educativo es orientado, teniendo como eje central el direccionamiento estratégico, la cultura y el clima institucional, así como el liderazgo en todos los procesos que se llevan a cabo, los cuales contribuyen al mejoramiento y fortalecimiento (Beltrán, 2017). En consecuencia, la gestión directiva es determinante para quienes la ejercen, pues ayuda a mantener el focoen los objetivos establecidos y en las tareas asignadas pararedireccionar, si hace falta,la administración de la organización (Beltrán, 2017).

\section{El clima organizacional y la convivencia}

Dentro de cualquier institución es sustancial que exista un buen clima organizacional y una convivencia armónica, factores clave para el desarrollo óptimo de la gestión directiva.Según Forehand y Gilmer (1964, citados por Sotelo y Figueroa, 2017), el clima organizacional es el conjunto de características percibidas por los trabajadores para describir a una organización y distinguirla de otras, de ahí que influya en el comportamiento de ellos. Por otra parte, Chiavenato (2006, citado por Pérez Maldonado, 2008) señala:

El clima organizacional se refiere al ambiente interno existente entre los miembros de la organización y está estrechamente ligado al grado de motivación de los empleados e indica de manera específica las propiedades motivacionales del ambiente organizacional, es decir, aquellos aspectos de la organización que desencadenan diversos tipos de motivación entre los miembros. Por consiguiente, es favorable cuando proporciona la satisfacción de las necesidades personales y la elevación de la moral de los miembros, y desfavorable cuando no logra satisfacer esas necesidades. El clima organizacional influye en el estado motivacional de las personas y viceversa (p. 28 ).

En concordancia con esta idea, Goncálvez (1997, citado por Pérez yRivera, 2015) describe el clima organizacional como la expresión personal de la percepción que los trabajadores y directivos se forman en la organización a la que pertenecen, lo cual incide directamente en su desempeño. Asimismo,Brow y Moberg (1990,citados por Pérez 


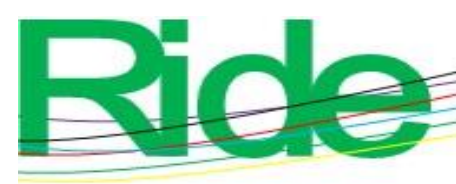

Revista lberoamericana para la

Investigación y el Desarrollo Educativo

ISSN 2007 - 7467

yRivera, 2015) consideran que el clima organizacional se refiere a una serie de características del medio ambiente interno organizacional tal y como lo perciben sus miembros. Otros autores, sin embargo, asocian el concepto clima organizacionalconclima institucional oclima escolar. Sandoval (2014), por ejemplo,se refiere al clima organizacional del siguiente modo:

El conjunto de características psicosociales de un centro educativo, determinados por aquellos factores o elementos estructurales, personales y funcionales de la institución, que, integrados en un proceso dinámico específico, confieren un peculiar estilo a dicho centro, condicionante, a la vez de los distintos procesos educativos (p. 169).

Por su parte, Arias (2009, citado por Carmonaet al., 2012) define el clima institucional en los siguientes términos:

El conjunto de interacciones y transacciones que se generan en la tarea educativa en una situación espacio-temporal. El clima tiene un carácter global y es determinado por elementos de naturaleza diversa como los espacios arquitectónicos, estructura física, equipamiento, características personales de los integrantes de la comunidad educativa, sistema de relaciones establecido, sistema de valores de distintos grupos entre otros factores, el clima institucional tiene un carácter dinámico e interactivo y es susceptible de modificación (p. 6).

Señalado lo anterior, se puede indicar que un clima organizacional adecuadoresulta vitalpara el óptimo funcionamiento de las instituciones educativas, "ya que este influye en el comportamiento de las personas en el trabajo y por supuesto en los resultados que se obtengan en determinada institución" (López et al., 2015, p. 15). En palabras de Pérez (2008), "la influencia que ejerce el clima organizacional en el rendimiento de cualquier institución, sea pública o privada, hacen que sea considerado un elemento imprescindible. Alcanzarlo debe constituir una preocupación básica de todos los niveles para conseguir los objetivos educativos" (p. 116).A estas ideas, Ruiz yPinchi(2016) añaden lo siguiente:

El clima organizacional es un factor preponderante para el desarrollo de las bases sentadas en una institución porque como consecuencia de un trabajo en equipo, la satisfacción laboral, tolerancia al conflicto, se forma un clima favorable y más aún si se entiende la promoción de acciones que colaboren a la autorrealización personal y profesional de sus miembros, las múltiples formas de relaciones sociales que se generan en la institución, en todos los 


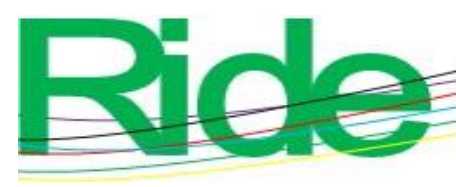

Revista Iberoamericana para la

Investigación y el Desarrollo Educativo

ISSN $2007-7467$

estamentos y se eligen y adecúa un estilo de gestión apropiado para la institución (p. 89).

Esto quiere decir que el clima organizacional se refiere al medio ambiente de trabajo, el cual puede ser o no percibido por los mismos integrantes de la organización. Esto, lógicamente, tiene un efecto en el comportamiento grupal e individual, lo que sirve para establecer diferenciasevidentes entre una organización y otra e incluso entre distintos departamentos de la misma empresa o institución(Pérez, 2008).

En la actualidad, cada vez existen más altos niveles de competitividad que exigen un mayor compromiso por parte de las instituciones hacia el cumplimiento de sus objetivos, lo cual demanda nuevas estrategias para la toma de decisiones y para dirigir al capital humano (Marín, Segredoy Perdomo, 2013), pues la calidad de este últimosuele condicionar los resultados que se puedan alcanzar. El capital humano, por ende,se puede definir como "todas aquellas habilidades y cualidades que la persona tiene al nacer, que acumula durante la vida y que contribuyen a que realice su trabajo de manera eficiente" (Vélez, Méndez y Vargas, 2016). Por ello, es queNavarro (2005) señala:

El capital humano es lo más importante de una organización debido al valor que generan las personas mediante la educación, la experiencia, la capacidad de conocer, de perfeccionarse, de tomar decisiones y de relacionarse con los demás (p. 4).

Igualmente, vale destacar sobre el capital humano que este "comprende la competencia, experiencia, conocimiento, valores y potencial innovador de los individuos dentro de la organización" (CalderónyMounsalli, 2012, p. 10),lo cual va "dirigido a la mejora de las capacidades del individuo y la capacidad de innovación de los grupos de trabajo” (Ramírez y Duván, 2007, p. 140). Aunado a esto, Mora (s.f., citado por Marínet al., 2013) destaca lo siguiente:

Con una buena administración del capital humano se impulsa su rendimiento y desarrollo y permite fomentar en las personas el establecimiento de metas y objetivos que impulsen el rendimiento de la organización y estimulen su compromiso para generar progresos en ella (p. 4). 


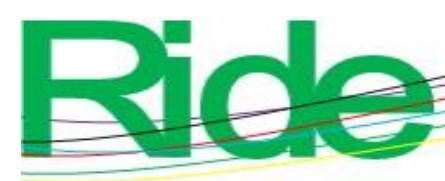

Revista Iberoamericana para la

Investigación y el Desarrollo Educativo

ISSN $2007-7467$

Ahora bien, dentro de las instituciones existe el denominado capital social, el cual surge de la interrelación entre los actores gubernamentales y la sociedad civil (Cabrera y García, 2018). Para Navarro (2005), el capital social se sustenta sobre señales y normas de contenido cultural, las cuales cooperan para generar un clima de confianza con base en un conjunto de constructos simbólicos que simplifican la interacción entre las personas. Dicho de otro modo, el capital social es un recurso que depende delos vínculosque tienen las personas consigo mismas y con las demás. Así pues, se puede afirmar que el capital social posee elementos que sirven para que una organización mejore su eficacia, confianza, normas y redes de asociación cívica, con la cuales se intentan promover iniciativas tomadas de común acuerdo entre sus miembros (Bolívar y Flores, 2011).

En definitiva, cuando se establece una armonía entreel capital humano, el capital social y el clima organizacional,los beneficios que se pueden alcanzar pueden ser superlativos, yaque — como señala Sandoval (2014) — la convivencia y el clima escolar juegan un rol fundamental en la gestión del conocimiento de los establecimientos educacionales. En concordancia con esto, Robinson (citado por Ulloa,Nail, Castro y Muñoz, 2012)explica que la mejora en los resultados de aprendizaje de los alumnos está fuertemente ligada al liderazgo. Esto significa que un liderazgo efectivo del director o directorase puede traducir en el establecimiento de metas y expectativas de aprendizaje compartidas con los docentes. Incuso puede favorecer la planificación, la coordinación y la evaluación de la enseñanza y el currículo, lo cual puede contribuir a la promoción y la participación del desarrollo docente.

La tarea de un líder institucional, entonces,se debe enfocar en promover un clima de colaboración. Al respecto, Grojean, Resick, Dickson y Smith (2004,citados por Riveros yGrimaldo,2017) afirman que los líderes no solo influyen directamente en el comportamiento de los miembros de una institución, sino que sus acciones también determinan las percepciones del clima de la organización.Por ello, dentro de las instituciones educativas el director se debe erigir como un líder con capacidades cognitivas y emocionales para impulsar las metas establecidas (Bolívar, 2011; Uribe, 2010). Para Loera (2003, citado por la Secretaría de Educación Pública [SEP], 2010) el liderazgo es concebido de la siguiente manera: 


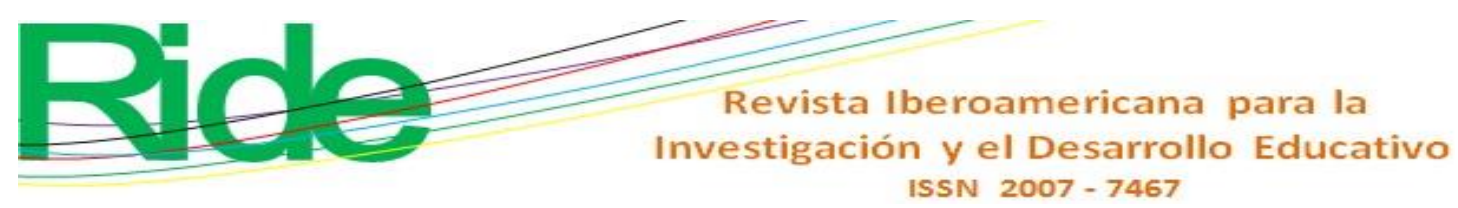

Capacidad de influir en las personas para que se esfuercen voluntaria y entusiastamente en el logro de las metas del grupo. Esta definición supone cuatro aspectos: 1) Capacidad para usar el poder (autoridad formalmente delegada por la institución) de modo responsable, 2) Capacidad para comprender que los seres humanos tenemos diferentes fuerzas de motivación en distintos momentos y situaciones, 3) Capacidad para inspirar (el objetivo) y 4) Capacidad para actuar en forma tal que se propicie un ambiente de respuesta a las motivaciones y fomento de estas (pp. 88-89).

Por ello,Finhum y Rhodes (2005, citados por Bell, Chan y Nel, 2014).) afirman que los líderes dan forma y desarrollan la realidad social de la organización para sus miembros, pues dan sentido a los valores y consiguen alcanzarla visión de la organización. Efectivamente, dentro de las instituciones, el director es el principal responsable de generar un clima de trabajo ameno y eficazpara potenciar el proyecto educativo,la eficacia de los profesores y los logros de aprendizaje de los estudiantes (Organización para la Cooperación y el Desarrollo Económicos [OECD], 2009).

El gran reto delos directores, en consecuencia, es cumplir con la misión educativa en conjunto con su equipo de trabajo; por ello,"se necesitan directores y equipos directivos escolares que cuenten con la preparación necesaria para generar modificaciones en la estructura de la organización educativa" (Marambio, 2016, p. 208).El líder, por tanto,además de delegar funciones administrativas, debe acompañarlas de un trabajo colaborativo que fomente el análisis colegiado de todos los actores de la comunidad educativa (Hernández, Tobón y Vázquez, 2015).

Rowe et al. (1998, citados por Azeska, Starc y Keveresky, 2017) manifiestan que los líderes deben de poseer visión y perspicacia para inspirar a otrosen la consecución delas metas trazadas.Como señalan Chiguay y Villagra (2016), "sin lugar a dudas quienes lideran las escuelas son agentes fundamentales en la mejora de la calidad de la educación” (p. 104), de ahí que - como apunta el Ministerio de Educación, Gobierno de Chile (s. f.)—deban ser capaces de impulsar las siguientes acciones:

- Promover los valores institucionales y un clima de confianza y colaboración para poder llegar al cumplimiento de las metas de la organización/institución.

- Fomentar un clima de colaboración entre el establecimiento, los estudiantes, padres y tutores. 
- Garantizar la articulación del proyecto educativo institucional con las características del contexto.

- Relacionarse con instituciones de su comunidad para potenciar el proyecto educativo institucional y los resultados de aprendizaje de los alumnos, generando redes de apoyo pertinentes.

- Informar a la comunidad y al sostenedor los logros y las necesidades del establecimiento educativo.

Por último,como destacan Ruiz y Pinchi (2016), es importante reiterar que el clima institucional se encuentra ligado con la gestión directiva.

Si en una institución educativa se desarrolla una cultura organizacional bien establecida y compartida por sus miembros, así como un clima institucional favorable ypositivo, solo de esa forma habría congruencia entre lo que se piensa, quiere y hace en una institución y el ambiente en el que se logran las metas institucionales (p. 89).

En pocas palabras, para que el gestor/líder de la institución pueda generar un clima organizacional y una convivencia amena, tiene que concentrase en desarrollar una óptima planificación estratégica para que la institución pueda funcionar eficientemente. En este sentido, Manes (2005) afirmaque unarespuesta oportuna a la necesidadde transformaciónen las instituciones educativasexige una planeación estratégica que contemple la condición holística y compleja de lagestión institucional, pues de esa manera se puede trazar elrumbo del mejoramiento de la calidad en la educación.

\section{Metodología}

EL presente trabajose sustentó en el paradigma cuantitativo, específicamente en el ámbito de la denominada investigación evaluativa(Bauselas, 2003). En estaconvergen la diversidad de perspectivas y los enfoques de la investigación debido a que supropósito final se concentra en transformar positivamente una condición asociada a un programa, proceso, institución,política, etc. Para ello, procura recabar información sobre un objeto de estudio para ofrecer propuestas de mejora, contribuir a la toma de decisiones o conocer de forma 


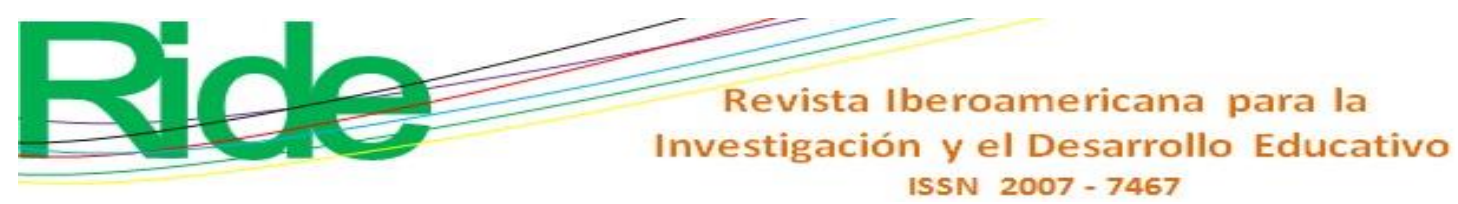

más precisa los problemas detectados.Ridde y Dagenais (2015) explican que la palabra clave en este análisis es transformación,pues el objetivo es conseguir la aplicación de un programa, proceso o política que se pueda desarrollar a corto y largo plazo.

Para el acercamiento empírico al objeto de estudio seutilizó un cuestionario asociado a la evaluación de la gestión directiva (diseñado originalmente por Sevilla, Medrano y Gaete), el cual forma parte del Modelo de Aseguramiento de la Calidad de la Gestión Escolar (SACGE) del Ministerio de Educación de Chile (2005). Este cuestionario fue diseñado mediante una escala de Likert, al cual se le aplicó el alfa de Cronbach,que arrojó un coeficiente alto de fiabilidad (0.959).

El cuestionario fue contestado por 2685 profesores (universo= 3480)decinco Subsistemas de Educación Media Superior del Estado de Baja California, México (Colegio de Bachilleres;Colegio de Estudios Científicos y Tecnológicos; Centro de Bachillerato Tecnológico, Industrial y de Servicios; Preparatoria Federal Lázaro Cárdenas; Colegio Nacional de Educación Profesional Técnica, y Centro de Bachillerato Técnico Agropecuario).La muestra fue calculada con la fórmula para poblaciones finitas, la cual arrojó $95 \%$ de confianza (Herrera, s.f.).

\section{Resultados}

En la tabla 1se exponen las apreciaciones de los profesores sobre aquellas acciones donde el director y el equipo directivo tienen gran relevancia parapropiciar el clima organizacional y convivenciafavorable en torno al proyecto educativo y a los logros de aprendizaje de los estudiantes. 
Tabla 1. Acciones directivas asociadas a la gestión del clima organizacional y la convivencia

\begin{tabular}{|l|c|c|c|}
\hline En esta institución... & $\begin{array}{c}\text { De } \\
\text { acuerdo } \\
\%\end{array}$ & $\begin{array}{c}\text { En } \\
\text { desacuerdo } \\
\%\end{array}$ & $\begin{array}{c}\text { No sabe } \\
\%\end{array}$ \\
\hline Se evalúa para mejorar. & 21 & 75.5 & 3.1 \\
\hline $\begin{array}{l}\text { Se generan planes institucionales para orientar la } \\
\text { acción. }\end{array}$ & 23.8 & 60 & 14.8 \\
\hline $\begin{array}{l}\text { Los resultados institucionales retroalimentan } \\
\text { nuevas acciones. }\end{array}$ & 25.4 & 65.9 & 7.8 \\
\hline Se consideran objetivos y metas del personal. & 39 & 52.6 & 7.6 \\
\hline Se incentiva a los mejores docentes. & 44.1 & 47.9 & 7.1 \\
\hline Se realizan proyectos equipo. & 32.8 & 50.3 & 6.0 \\
\hline $\begin{array}{l}\text { Participan activamente padres, alumnos, profesores } \\
\text { y coordinadores. }\end{array}$ & 36.3 & 56.8 & 6.0 \\
\hline $\begin{array}{l}\text { Los reglamentos regulan las relaciones entre el } \\
\text { personal. }\end{array}$ & 29.2 & 66.9 & 3.2 \\
\hline $\begin{array}{l}\text { Los directivos son promotores del buen clima de } \\
\text { trabajo. }\end{array}$ & 29 & 69 & 1.3 \\
\hline $\begin{array}{l}\text { Se muestra satisfacción con los resultadosde los } \\
\text { estudiantes. }\end{array}$ & 18.4 & 78.4 & 2.6 \\
\hline Laresolución de conflictoses justa y expedita. & 37.8 & 56.7 & 5.0 \\
\hline
\end{tabular}

Fuente: Elaboración propia

Como se puede observar en la tabla 1, los profesores están mayoritariamente endesacuerdo con la formaen que los directivos realizan sus funciones, especialmente en lo concerniente a la reducida satisfacción con los resultados de los estudiantes y al uso inadecuado de las acciones de evaluación. De hecho, se puede evidenciar que, según sus percepciones, los directivos no necesariamente son promotores del buen clima de trabajo.

A continuación, en la tabla 2, se identifica el grado de instalación de los sistemas de gestión del clima organizacional y la convivencia por parte de los directivos institucionales. Es esta tablase destaca la atención hacia el propio proyecto educativo institucional y la consabida posibilidad de generar redes de apoyo para la propia institución. 
Tabla 2. Grado de instalaciones de los sistemas de gestión del clima organizacional y la convivencia

\begin{tabular}{|l|c|c|c|}
\hline En esta institución... & $\begin{array}{c}\text { De } \\
\text { acuerdo } \\
\%\end{array}$ & $\begin{array}{c}\text { En } \\
\text { desacuerdo } \\
\%\end{array}$ & $\begin{array}{c}\text { No sabe } \\
\%\end{array}$ \\
\hline $\begin{array}{l}\text { El director y equipo directivo promueven un clima } \\
\text { de colaboración entre la escuela, los estudiantesy } \\
\text { los padres. }\end{array}$ & 31 & 50 & 17.4 \\
\hline $\begin{array}{l}\text { El director y equipo directivo promueven los } \\
\text { valores institucionales y un clima de confianza y } \\
\text { colaboraciónpara el logro de sus metas. }\end{array}$ & 30.9 & 55.5 & 12.8 \\
\hline $\begin{array}{l}\text { El director y equipo directivo transforman los } \\
\text { resultados de los procesos de evaluaciónen } \\
\text { prioridades de mejoray oportunidades de } \\
\text { innovaciónpedagógica. }\end{array}$ & 30.7 & 55.8 & 12.7 \\
\hline $\begin{array}{l}\text { El director y equipo directivo utilizan los resultados } \\
\text { de los procesos de evaluaciónen oportunidades para } \\
\text { el aprendizaje organizacional. }\end{array}$ & 31.7 & 52.8 & 14.8 \\
\hline $\begin{array}{l}\text { El director y equipo directivo garantizan la } \\
\text { articulación de la definición del proyecto educativo } \\
\text { institucional con las características del entorno. }\end{array}$ & 27.4 & 56.6 & 15.2 \\
\hline $\begin{array}{l}\text { El director y equipo directivo se relacionan con } \\
\text { instituciones de su comunidad para potenciar el } \\
\text { proyecto educativo institucional y los resultados de } \\
\text { aprendizaje de los estudiantes, generando redes de } \\
\text { apoyo pertinentes. }\end{array}$ & 34.8 & 55.2 & 8.0 \\
\hline
\end{tabular}

Fuente: Elaboración propia

En la tabla 2 se aprecia que el sistema de gestión visualizado con mayores deficiencias se relaciona con la poca articulación entre el proyecto educativo institucional y las necesidades y características de su entorno. Igualmente, sobresale la percepción negativa sobre los resultados de los procesos de evaluación en prioridades para mejorar e innovar lo pedagógico.

Por último, en la tabla 3 se expone la apreciación de los profesores en relación con los procesos que caracterizan la gestión del buen clima organizacional y la convivencia por parte de los directivos institucionales. 


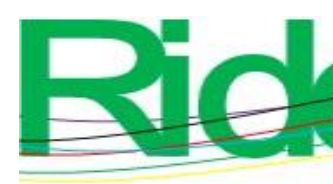

Revista Iberoamericana para la

Investigación y el Desarrollo Educativo

ISSN 2007 - 7467

Tabla 3. Grado de mejoría que requiere la gestión del clima organizacional y la convivencia

\begin{tabular}{|l|c|c|c|}
\hline En esta institución... & $\begin{array}{c}\text { De } \\
\text { acuerdo } \\
\%\end{array}$ & $\begin{array}{c}\text { En } \\
\text { desacuerdo } \\
\text { \% }\end{array}$ & $\begin{array}{c}\text { No sabe } \\
\text { \% }\end{array}$ \\
\hline $\begin{array}{l}\text { Está orientada hacia los alumnos, sus familias y la } \\
\text { comunidad: la atención que la institución les brinda } \\
\text { y la forma en que los incorpora en sus actividades. }\end{array}$ & 54 & 39.8 & 5.5 \\
\hline $\begin{array}{l}\text { La forma en que el director y el equipo directivo } \\
\text { conducen la institución. }\end{array}$ & 49.8 & 36.8 & 12.4 \\
\hline
\end{tabular}

Fuente: Elaboración propia

En la tabla 3 se aprecia la deficiente atención que brinda la institución educativa a la comunidad, pues esta última difícilmente aparece considerada en la planeación institucional o en las acciones cotidianas. Asimismo, $49.8 \%$ de los profesores sugieren que debe mejorar mucho la forma en que el director y los equipos directivos conducen la institución.

\section{Discusión}

De acuerdo con los resultados expuestos en esta investigación, la función de la gestión directiva en las instituciones educativas no debe de perder de vista el que los estudiantes obtengan resultados académicos satisfactorios (Araiza et al., 2014). Por ello, resulta vital que la persona que asuma el liderazgo formal en una institución educativa del nivel medio superior fomente la construcción del tejido humano de sus escuelas (Antúnez, 1993, citado por INEE, s.f.)para cumplir las metas planteadascomo comunidad educativa, especialmente en esta época donde la tarea de un director escolar se ha complejizado por múltiples factores.

La función directiva, por ende, debe de potenciar la gestión de la mejora del clima organizacional y la convivencia dentro de los planteles de la educación media superior, para lo cual se debe trabajar de manera constante en la construcción de un perfil que se ajuste a los retos y las demandas deese nivel educativo.

Por otra parte, y según los resultados presentados en este estudio, se debe considerar que los profesores consultados están mayoritariamente endesacuerdo, en especial, con la reducida satisfacción que los directivos muestran con los resultados de los estudiantes y con el uso inadecuado de las acciones de evaluación. Lo anterior, sin duda, debe de ser materia 


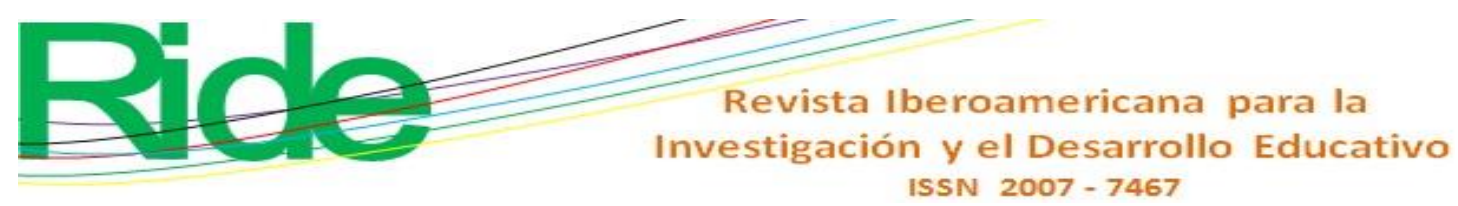

de reflexión para replantear la manera en que los directivos se forman en esta área, pues esta constituye una labor de vital importancia para potenciar los aprendizajes delos alumnos, así como la organización de la comunidad educativa.

\section{Conclusiones}

La panorámica explorada en este estudioenseña la importancia de la gestión que los directores y sus equipos directivos deben asumir para avanzar positivamente en la construcción de espacios formativos que se caractericen por su flexibilidad para integrar equipos de trabajo que puedan encarar los retos y las metas establecidas. Igualmente, se debe recordar que las responsabilidades de este profesional involucran no solo el fomento de las prácticas escolares y pedagógicas, sino también el impulso de estrechos vínculos armoniosos con la comunidad, y no conflictivos o distantes, como lo han señalado los participantes de esta investigación.

Asimismo, no es suficiente que los directivos presenten novedosos planes de desarrollo institucional si estos no son comunicados en un marco de horizontalidad, respeto y aceptación de las experiencias y expectativas de quienes participan en la institución educativa. De hecho, se deben aprovecharlas potencialidades de todospara evitar caer en la complacencia individual. Por ello, los directivos deben considerar que las instituciones que administran son parte de una comunidad, la cual, en definitiva, es su verdadera "propietaria", porque tiene unas expectativas y necesidades que deben ser tomadas en cuenta para impulsar su progreso socioeconómico. 


\section{Referencias}

Araiza, S., Magaña y García, R. y Carrillo, L. E. (2014). Evaluación por estándares de la gestión directiva en secundaria. Revista Iberoamericana de Educación, 64, 99-113. Recuperado de https://rieoei.org/RIE/article/view/408.

Azeska, M. A., Starc, J. and Keveresky, L. (2017). Styles of decision making and management and dimensions of personality of school principals. International Journal of Cognitive Research in Science, Engineering and Education, 5(2), 47-56. Doi: 10.5937/IJCRSEE1702047A

Bauselas, E. (2003). Metodología de la investigación evaluativa: Modelo CIPP. Revista Complutense de Educación, 14(2), 361-376. Recuperado de http://revistas.ucm.es/index.php/RCED/article/view/RCED0303220361A/16386.

Bell, C., Chan, M. and Nel, P. (2014). The Impact of Participative and Directive Leadership on Organisational Culture: An Organisational Development Perspective. Mediterranean Journal of Social Sciences, 5(23), 1970-1985. Retrieved from http://www.mcser.org/journal/index.php/mjss/article/viewFile/4744/4602.

Beltrán, S. M. (2017). La gestión directiva: un concepto construido desde las comprensiones de los directivos docentes de las escuelas públicas bogotanas. Revista Iberoamericana para la Investigación y el Desarrollo Educativo, 7(13), 128. http://www.scielo.org.mx/scielo.php?script=sci_arttext\&pid=S200774672016000200562\&lang=es.

Bolívar, G.A., Flores L. (2011) Discutir el campo del capital social desde un enfoque transdisciplinario. $29 . \quad$ Polis, Recuperado de: http://journals.openedition.org/polis/1911

Bolívar, A. (2011). Aprender a liderar líderes. Competencias para un liderazgo directivo que promueva el liderazgo docente. Educar, 47(2), 253-275. Recuperado de https://www.raco.cat/index.php/Educar/article/view/248536/332663. 
Cabrera, M. F. y García, L. S. (2018). Medición del capital social en la educación superior: una alternativa frente a la disyuntiva del desarrollo. Sophia, 14(1), 12-21. Doi: http://dx.doi.org/10.18634/sophiaj.14v.1i.490

Calderón, J. y Mounsalli, G. (2012). Capital humano: elemento de diferenciación de las organizaciones. Actualidad Contable Faces, 15(24), 5-18. Recuperado de http://www.redalyc.org/articulo.oa?id=25722697002.

Carmona, O., Flores, P., Gómez, W., Navas, M., Ruiz, M. y Smith, K. (2012). El clima institucional como generador de contextos de protección y riesgo frente a las manifestaciones de violencia en centros educativos del Circuito 06 de la Dirección Regional de Educación de San José. Revista Gestión de la Educación, Escuela de Administración Educativa, 2(1), 1-28.

Chiguay López, M. S., \& Villagra Bravo, C. P. (2016). Evaluación interna ascendente del desempeño directivo: una experiencia de gestión y liderazgo escolar. Gestión de la Educación, 6(2), 103-117. Doi:http://dx.doi.org/10.15517/rge.v1i2.25491

Cruzata, A. y Rodríguez, I. E. (2016). La gestión en las instituciones educativas: enfoques, modelos y posiciones teóricas y prácticas. Revista de Gobierno y Gestión Pública, 3(10), 1-10. Recuperado de http://www.revistagobiernoydegestionpublica.com/index.php/REVIGGP/article/vie $\underline{\mathrm{w} / 31 / 47}$

Hernández, J. S., Tobón, S. y Vázquez, J. M. (2015). Estudio del liderazgo socioformativo mediante la cartografía conceptual. Revista Iberoaméricana de Evaluación Educativa, $\quad 8(2), \quad$ 105-128. Recuperado de https://revistas.uam.es/index.php/riee/article/view/2878.

Herrera, M. (s. f.) Fórmula para calcular muestra en poblaciones finitas. Recuperado de https://investigacionpediahr.files.wordpress.com/2011/01/formula-para-cc3a1lculode-la-muestra-poblaciones-finitas-var-categorica.pdf.

Instituto Nacional para la Evaluación de la Educación [INEE] (s. f.). Capítulo 1. La función directiva, su importancia y sus características. Recuperado de http://www.inee.edu.mx/images/stories/lafunciondirectiva/capitulo\%201.pdf. 


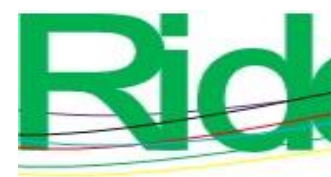

Revista lberoamericana para la

Investigación y el Desarrollo Educativo

ISSN $2007-7467$

López, E., Espinoza, S., Serna, A., Ponce, S., Galván, M., Oviedo, A., Montoya, Y., Lizárraga, A., Hilario, M., Ibarra, M., Valverde, M., Cuadras, V., Cháires, M. y Gómez, J. (2015). Evaluación de la instrumentación de la reforma de la educación media superior. Colombia: Redipe.

Manes , J. (2005). Gestión estratégica para instituciones educativas: guía para planificar estrategias de gerenciamiento institucional. Buenos Aires-México-SantiagoMontevideo: Granica.

Marambio, C. A. (2016). Experiencia de gestión educativa escolar. Una acción dinámica para la transformación cultural. Educar, 54(1), 205-226. Doi: https://doi.org/10.5565/rev/educar.809

Marín, X., Segredo, A. M. y Perdomo, C. I. (2013). Capital humano, gestión académica y desarrollo organizacional. Educación Médica Superior, 27(3), 1-10. Recuperado de http://ems.sld.cu/index.php/ems/rt/printerFriendly/231/117.

Ministerio de Educación, Gobierno de Chile (s. f.). Ministerio de Educación, Gobierno de Chile.

Recuperado dehttp://www.gestionyliderazgoeducativo.cl/gestioncalidad/buenadireccion/default. php.

Navarro, I. (2005). Capital humano: su definición y alcances en el desarrollo local y regional. Archivos Analíticos de Políticas Educativas, 13(35), 1-36. Recuperado de http://www.redalyc.org/articulo.oa?id=275020513035.

Organización para la Cooperación y el Desarrollo Económicos [OECD] (2009). Leading to Learn: School Leadership and Management Styles. OECD. Recuperado de https://www.oecd.org/berlin/43541674.pdf.

Pérez, E. A. (2008). Análisis del clima organizacional en la Facultad de Ciencias Administrativas de la Universidad Autónoma de Baja California (tesis de doctorado). Mexicali, Baja California, México. Recuperado de https://drive.google.com/file/d/15QxViCddGDFpg0d9DaHuV8C9HVCQzcFo/view

Pérez Maldonado, E. A. (2008). Análisis del clima organizacional en la facultad de Ciencias Administrativas de la Universidad Autónoma de Baja California, unidad Mexicali (tesis de maestría). Universidad Autónoma de Baja California. Mexicali, 


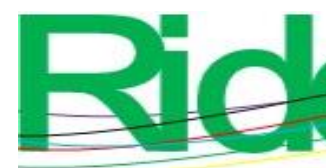

Baja

California,

Revista lberoamericana para la

Investigación y el Desarrollo Educativo ISSN $2007-7467$

https://drive.google.com/file/d/15QxViCddGDFpg0d9DaHuV8C9HVCQzcFo/view

Pérez, N. O. y Rivera, P. L. (2015). Clima organizacional y satisfacción laboral en los trabajadores del Instituto de Investigaciones de la Amazonía Peruana, período 2013(tesis de maestría). Iquitos, Perú. Recuperado de http://repositorio.unapiquitos.edu.pe/handle/UNAP/3810

Poggi, M. (2001). La formación de directivos de instituciones educativas. Algunos aportes para el diseño de estrategias. Buenos Aires: Instituto Internacional de Planeamiento de la Educación IIPE/UNESCO.

Ramírez, O. y Duván, E. (2007). Capital intelectual. Algunas reflexiones sobre su importancia en las organizaciones. Pensamiento y Gestión,(23), 131-152. Recuperado de http://www.redalyc.org/articulo.oa?id=64602306.

Ridde, V. y Dagenais, Ch. (2015). Enfoques y prácticas en la evaluación de programas. Colombia. Editorial Pontificia Universidad Javeriana.

Riveros, P. N. y Grimaldo, M. P. (2017). Valores y clima organizacional en docentes de un instituto de educación superior en Lima. Ciencias Psicológicas, 11(2), 179-188. Doi: $10.22235 / c p . v 11 i 2.1489$

Ruiz, C. y Pinchi, W. (2016). Impacto en la cultura organizacional en la gestión educativa de la Unidad de Gestión Educativa Local de Ascope, 2014 (tesis de doctorado). Trujillo, Perú: Universidad Nacional de Trujillo.

Sandoval, M. (2014). Convivencia y clima escolar: claves de la gestión del conocimiento. Ultima Década, 22(41), 153-178. Recuperado de https://scielo.conicyt.cl/pdf/udecada/v22n41/art07.pdf

Sandoval, M., Surdez, E. y Pérez, A. (2017). Clima escolar del campus de ingienería y arquitectura de una universidad pública mexicana desde la perspectiva de sus estudiantes. Revista Electrónica Educare, 21(2), 1-21. Doi: http://dx.doi.org/10.15359/ree.21-2.8

Secretaría de Educación Pública [SEP] (2010). Modelo de gestión educativa estratégica. México, D.F. 


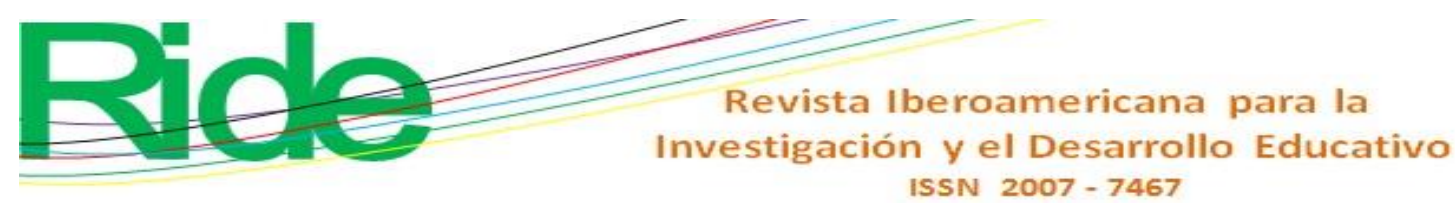

Sotelo, J. G. y Figueroa, E. G. (2017). El clima organizacional y su correlación con la calidad en el servicio en una institución de educación de nivel medio superior. Revista Iberoamericana para la Investigación y el Desarrollo Educativo, 8(15), 128. Doi: 10.23913/ride.v8i15.312

Ulloa, J., Nail, O., Castro, A. y Muñoz, M. (2012). Problemas de gestión asociados al liderazgo como función directiva. Estudios Pedagógicos, 38(1), 121-129. Recuperado de https://scielo.conicyt.cl/pdf/estped/v38n1/art07.pdf

Uribe, M. (2010). Profesionalizar la dirección escolar potenciando el liderazgo: una clave ineludible en la mejora escolar. Desarrollo de perfiles de competencias directivas en el Sistema Educativo Chileno. Revista Iberoamericana de Evaluación Educativa, 3(1e), 303-322. Recuperado de http://www.rinace.net/riee/numeros/vol3num1_e/art22.pdf

Vázquez, S., Liesa, M. y Bernal, J. L. (2016). El camino hacia la profesionalización de la función directiva: el perfil competencial y la formación del director de centros educativos de España. Perfiles Educativos, 48(151), 158-174.

Vélez, S., Méndez, V. y Vargas, L. (2016). Familia, capital humano y psicología industrial/organizacional. Interamerican Journal of Psychology,50(3), 433-440.

Weinstein, J., Hernández, M., Cuellar, C. y Flessa, J. (2014). Liderazgo escolar en América Latina y el Caribe. Experiencias innovadoras de formación de directivos escolares en la región. Chile: Oficina Regional de Educación para América Latina y el Caribe OREALC/UNESCO. 
Revista Iberoamericana para la

Investigación y el Desarrollo Educativo

ISSN 2007 - 7467

\begin{tabular}{|c|c|}
\hline Rol de Contribución & Autor (es) \\
\hline Conceptualización & $\begin{array}{l}\text { Evangelina López, Luis Fernando García Hernández y Jorge Eduardo Martínez } \\
\text { "igual” }\end{array}$ \\
\hline Metodología & Evangelina López "principal” \\
\hline Software & NO APLICA \\
\hline Validación & Evangelina López "principal” \\
\hline Análisis Formal & $\begin{array}{l}\text { Evangelina López "igual” Luis Fernando García Hernández "igual” Jorge Eduardo } \\
\text { Martínez "igual” }\end{array}$ \\
\hline Investigación & Evangelina López "principal” \\
\hline Recursos & Evangelina López “principal” \\
\hline Curación de datos & Evangelina López "principal” \\
\hline $\begin{array}{l}\text { Escritura - Preparación del borrador } \\
\text { original }\end{array}$ & $\begin{array}{l}\text { Evangelina López "igual” Luis Fernando García Hernández "igual” Jorge Eduardo } \\
\text { Martínez "igual” }\end{array}$ \\
\hline Escritura - Revisión y edición & $\begin{array}{l}\text { Evangelina López “principal” Luis Fernando García Hernández "que apoya” Jorge } \\
\text { Eduardo Martínez "que apoya” }\end{array}$ \\
\hline Visualización & Evangelina López, Luis Fernando García Hernández Jorge Eduardo Martínez \\
\hline Supervisión & Evangelina López "principal” \\
\hline Administración de Proyectos & Evangelina López "principal" \\
\hline Adquisición de fondos & Evangelina López "principal” \\
\hline
\end{tabular}

